\title{
Retail Bankruptcy Prediction
}

\author{
${ }^{1}$ Johnny Pang and ${ }^{2}$ Mark Kogel \\ ${ }^{1}$ Department of Mathematics, Monmouth University, West Long Branch, New Jersey, USA \\ ${ }^{2}$ Department of Finance, Monmouth University, West Long Branch, New Jersey, USA
}

Received 2013-06-11, Revised 2013-09-09; Accepted 2013-09-13

\begin{abstract}
This study reintroduces the famous discriminant functions from Edward Altman and Begley, Ming and Watts (BMW) that were used to predict bankrupts. We will formulate three new discriminant functions which differ from Altman's and BMW's re-estimated Altman model. Altman's models as well as Begley, Ming and Watts's re-estimated Altman model apply to publicly traded industries, whereas the new models formulated in this study are based on retail companies. The three new functions will provide better predictions on retail bankruptcy and they will minimize the chance of misclassifications.
\end{abstract}

Keywords: Multiple Discriminant Analysis, MDA, Altman's z Score, BMW's z Score, Retail, Bankruptcy

\section{INTRODUCTION}

Bankruptcy has been known to have an effect on a firm's managers, employees, suppliers, investors, customers and communities. It would be beneficial and important to be able to predict the likelihood of bankruptcy so that actions could be implemented to avoid it or prevent it from actually happening. Ratio analysis is one technique used to analyze a firm's financial health and could be used to foresee future bankruptcy. However, Multiple Discriminant Analysis (MDA) has been a popular technique to predict bankruptcy in recent years. Multiple discriminant analysis is a similar statistical regression technique that uses financial ratios as explanatory variables to predict a qualitative variable, whether the firm goes bankrupt or stays solvent. Edward Altman is one of the most known innovators of predict bankruptcy using MDA. Corporations, banks and credit analysts can use the Altman z-score model to predict the probability of future solvency and bankruptcy. Regulators are also concerned with bankruptcy and use these models. Altman has developed two discriminant functions to predict bankruptcy for publicly traded firms. His original z-score model was used to predict bankruptcy for publicly traded manufacturing firms and the other (his revised model) was used for publicly traded non-manufacturing firms. Begley, Ming and Watts were also innovators of MDA and used the same explanatory variables in Altman's original model. However, they used financial data from the 1980's to come up with their own weights for their re-estimated Altman model (Begley et al., 1996).

Our goal is to formulate discriminant models that are specific to only the retail industry and that can better model bankruptcy prediction in the retail. The retail industry consists of only restaurants, discount stores, building supply stores, whole sale food stores, automotive and retail hardlines and softlines. It should be noted that the retail industry is a very important industry to the US economy. According to Plunkett Research, 1td, the retail industry, with more than 14 million employees in America alone (about one out of 10 workers), is one of the largest industries in the world by number of businesses and number of employees. In 2011, the retail industry generated over $\$ 4.7$ trillion dollars in revenues, making it one of the most largest and important industries in the United States economy. Since the retail industry is so vital to the US economy, it is necessary that there should be specific discriminant functions that can foresee bankruptcy.

\section{LITERATURE REVIEW}

\subsection{Altman's Z Score}

As mentioned above, Altman was an innovator in bankruptcy prediction using MDA. It is necessary to Corresponding Author: Johnny Pang, Department of Mathematics, Monmouth University, West Long Branch, New Jersey, USA 
mention his previous work in the field of bankruptcy prediction. Altman wrote his first study on bankruptcy prediction in 1968 titled Financial Ratios, Discriminant Analysis and the Prediction of Corporate Bankruptcy. Altman sampled sixty six firms of the sixty six firms thirty three were bankrupt and the other thirty three were solvent manufacturing firms. These firms were used to establish a function which best discriminates between companies in two mutually exclusive groups: bankrupt and nonbankrupt firms. The discriminant function predicts a $\mathrm{z}-$ score that tells the analyst the probability of default. Altman collected balance sheet and income statement data on the sixty six firms analyzed and from that data computed twenty two financial ratios of the twenty two financial ratios, the most significant five were selected as the explanatory variables for the discriminant function. The best five variables that were selected were net working capital to total assets ratio, earnings before interest and taxes to total assets ratio, retained earnings to total assets ratio, market value of equity to total liabilities ratio and finally sales to assets ratio. Below lists the original Altman z-score equation with zones of discrimination and explanation of explanatory variables Equation 1:

$$
\mathrm{Z}=1.2 \mathrm{X}_{1}+1.4 \mathrm{X}_{2}+3.3 \mathrm{X}_{3}+0.6 \mathrm{X}_{4}+1.0 \mathrm{X}_{5}
$$

Assuming that the zones of discrimination are:

$$
\begin{aligned}
& \mathrm{Z}<1.81 \text {-- Distress } \\
& 1.81<\mathrm{Z}<2.99 \text {--Inconclusive } \\
& \mathrm{Z}>2.99 \text {--Solvent }
\end{aligned}
$$

$\mathrm{X}_{1}=$ The ratio of working capital to total assets. This is a measure of the net liquid assets of a firm relative to capitalization. Working Capital is calculated by subtracting the firm's current assets from current liabilities.

$\mathrm{X}_{2}=$ The ratio of retained earnings to total assets. This measures a firm's cumulative profits relative to size. When a firm generates a profit, some of the profit is distributed to the shareholders as dividends and the rest is accumulated to the balance sheet in an account in the equity section. These retained earnings are used to pay off debt or invest in research and development. The age of the firm is implicitly considered due to the fact that relatively young firms have a lower ratio and the incidence of business failures is much higher in a firm's early years. It is also an indication of the firm's use of external capital to fund its investments and operations.

$\mathrm{X}_{3}=$ The ratio of earnings before interest and taxes to total assets. This is a measure of the earning power of the firm's assets without any influence from tax or leverage factors.

$\mathrm{X}_{4}=$ The market value of equity to total liabilities ratio. This measures the extent to which a firm's assets can decline in value before book value becomes negative and the firm becomes insolvent. This assesses the ability of a firm to fund its operations with equity capital, the cost of equity and the market's outlook for the firm's prospects. This also adds a market basis dimension to the calculation. Market Capitalization is another name for market value of equity and is calculated by multiplying the company's stock price by the total amount of shares outstanding.

$\mathrm{X}_{5}=$ The ratio of sales to total assets. This is a turnover ratio that measures the sales generating capacity of the firm's assets.

After finding the optimal weights of the five explanatory financial ratio variables, Altman ran a series of test to see how accurate the model was. He found that "72 percent correct assignment is evidence that bankruptcy can be predicted two years prior to the event (Altman, 2000)".

The Altman z-score normally range from -5.0 to +20.0 , although higher scores may occur if a company has a high equity value and/or low level of liabilities. Scores above 3.0 indicate bankruptcy is unlikely, scores between 1.81 and 2.99 are inconclusive and scores below 1.81 indicate an increased risk of business failure. The best cutoff value that Altman found was a z-score of 2.675 because the interval between scores 2.67 and 2.68 had the least number of misclassified firms out of all of the $\mathrm{z}$ score intervals within the zone of ignorance interval Altman (1968). So for instance, if a firm were to fall in the ignorance interval that was below 2.675, an analyst could classify it as bankrupt in the near future. This $\mathrm{z}$ score "best discriminates between the bankrupt and non bankrupt firms (Altman, 2000)". In general, the higher the $\mathrm{z}$-score, the better the credit worthiness of the firm. A z-score below zero indicates an extremely risky situation.

\subsection{Altman's Revised Z Score}

Altman also introduced a revised z-score model that catered towards publicly traded non-manufacturing firms in his study titled, Predicting Financial Distress of Companies: Revisiting the Z Score and ZETA $®$ Models (2000). The new model is stated below with zones of discrimination Equation 2:

$Z^{\prime \prime}=6.56 \frac{\mathrm{NWC}}{\mathrm{TA}}+3.26 \frac{\mathrm{RE}}{\mathrm{TA}}+6.72 \frac{\mathrm{EBIT}}{\mathrm{TA}}+1.05 \frac{\mathrm{BVE}}{\mathrm{TL}}$

Assuming the zones of discrimination is: 


$$
\begin{aligned}
& Z<1.1 \text {-- Distress } \\
& 1.1<Z<2.6 \text {-- Grey Area } \\
& Z>2.6 \text {-- Solvent }
\end{aligned}
$$

There are only four explanatory variables in this model compared to Altman's original z-score function. The fourth explanatory variable used in this formula is book value of equity to total liabilities ratio. The original used market value of equity instead of book value of equity to total liabilities. Also, sales to assets ratio is not included in this model because including it increased the likelihood of missing a potential bankruptcy for nonmanufacturing firms. The revised Altman z-score cannot be used for publicly traded and privately held manufacturing companies. This model could be good to use for retail firms.

\subsection{Begley, Ming and Watts' Model}

Begley, Ming and Watts used the same five ratios that Altman used in his original discriminant model. However, they used a larger data sample of one hundred random non-bankrupt firms and one hundred random bankrupt firms in the 1980's. Begley used the same discriminant zones and cutoff off point of 2.675 as Altman. Equation 4 shows the re-estimated Altman model below Equation 3:

$$
\begin{aligned}
& \mathrm{Z}=10.4\left(\frac{\mathrm{NWC}}{\mathrm{TA}}\right)+1\left(\frac{\mathrm{RE}}{\mathrm{TA}}\right)+10.6\left(\frac{\mathrm{EBIT}}{\mathrm{TA}}\right) \\
& +0.3\left(\frac{\mathrm{MVE}}{\mathrm{TL}}\right)-0.17\left(\frac{\mathrm{Sales}}{\mathrm{TA}}\right)
\end{aligned}
$$

The re-estimated Altman model has different coefficients than Altman's original model, especially the MVE/TL coefficient. Altman's MVE/TL coefficient is two times as large as the new MVE/TL coefficient. According the Begley, Ming and Watts, a lower MVE/TL coefficient indicates that "a small reduction in debt has a smaller effect on the firms z-score than it did before, consistent with the notion that the probability of bankruptcy is less sensitive to changes in the level of debt carried by the firm than it was in the pre-1980 years (1996)".

\section{COMPARISONS}

Balance sheet and income statement data of forty randomly chosen publicly traded retail firms was gathered. Of the forty, eighteen were listed as bankrupt and twenty two were solvent. These retail firms consisted of retail hard and soft line firms, restaurants, food stores, discount stores, automotive stores and building supply stores. The financial data for bankrupt retail firms was retrieved from EDGAR Company Search. For the sample of bankrupt companies, the data are derived from financial statements dated one annual reporting period prior to bankruptcy. The most recent data for bankrupt companies will predict bankruptcy better one year in the future because the previous year contains the company's most recent and worst financial position in the company's lifetime. The model will assume that the firm's financial position in the current year, will get worse and probability of bankruptcy will increase in the next year. The financial data for solvent firms was retrieved from Charles Schwab and the financial data that was obtained from this group was from their most recent year that they reported. Table 1 presents each of the solvent retail firm's original and re-estimated BMW z-scores. Table 2 presents each of the bankrupt retail firm's original and BMW z-scores. The revised $\mathrm{z}$-scores of solvent and bankrupt retail firms are located in Table 3 and 4, respectively.

The above results show that the original Altman $\mathrm{z}$ function produced two grey area $\mathrm{z}$ scores and one completely wrong prediction. J.C. Penney, which is solvent, was classified as inconclusive because its ratios were lower than the average solvent ratios as can be seen below in Fig. 1. Movie Gallery, which is a bankrupt retail company, was the second firm that that the original Altman model classified as inconclusive. Reasons being is because Movie Gallery had a high EBIT/TA ratio of $8 \%$ while the bankrupt average was $-13 \%$.

Table 2 also showed the original Altman model misclassifying the bankrupt firm, The Penn Traffic Company as Solvent with a $\mathrm{z}$ score of 3.77. The reason for this misclassification was because it had a high SALES/TA ratio of 4.5 compared to the average of 2.2.

The BMW model misclassified Samsonite, a bankrupt firm as inconclusive with a high z-score of 2.35 because it had a high EBIT/TA ratio of $12.2 \%$ while the bankrupt average was $-13 \%$. Most of the coefficient weight in the BMW model is distributed towards the EBIT/TA ratio with a weight of 10.6. On the other hand, the revised model predicted all $\mathrm{z}$ scores correctly. Table 5 listed below shows some 
descriptive statistics of the original and revised zscores for both bankrupt and solvent companies. By reading the min and max values, one can see that the revised z-score does predict bankruptcy better than the original. It can be used for retail firms effectively.

After the z-scores were calculated for the twenty two solvent and eighteen bankrupt retail firms, a second test was conducted using the BMW model, original and the revised Altman models again on fifteen extra retail companies to test their accuracy. This time all of the models came up with wrong predictions. The BMW model predicted Wal-Mart and Costco as inconclusive. These z-scores were also below Altman's cutoff of 2.675 , which could classify these firms as bankrupt companies. Sears was classified as bankrupt with a score of -0.387 .

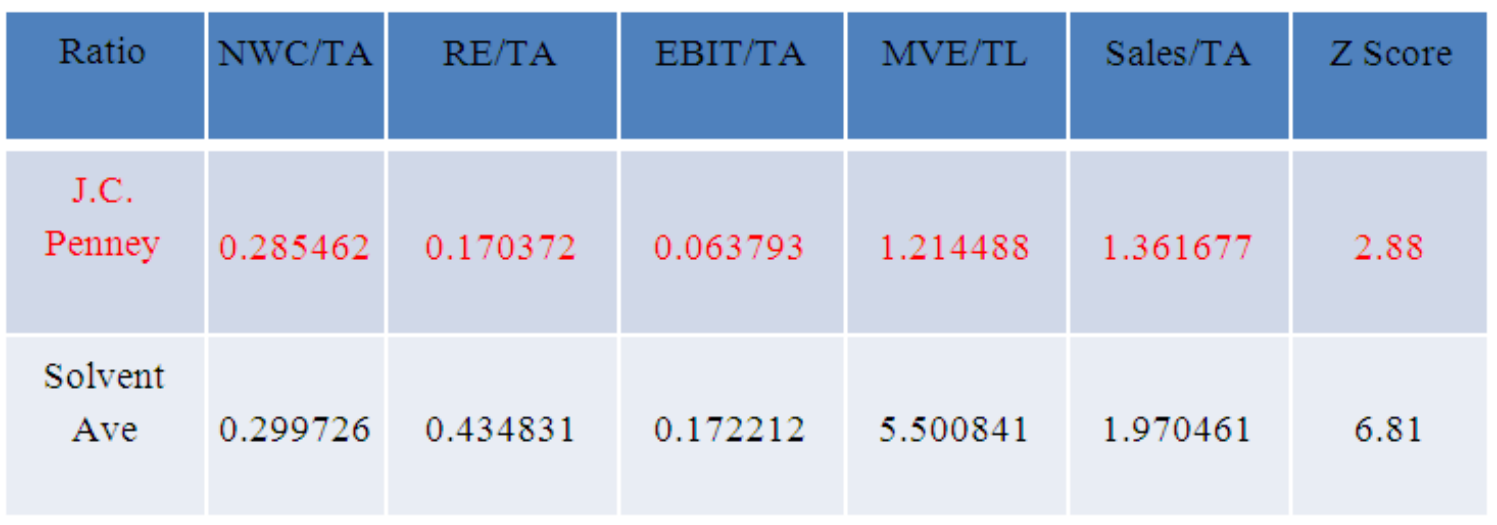

Fig. 1. Misclassifications from the Original Altman Z Model

Table 1. Solvent Firms Original Z-Scores and BMW Z-Scores

\begin{tabular}{|c|c|c|c|c|c|c|c|}
\hline Solvent & NWC/TA & $\mathrm{RE} / \mathrm{TA}$ & EBIT/ TA & MVE/TL & Sales/ TA & Z Score & BMW \\
\hline Aaron's, inc & 0.753 & 0.754 & 0.42 & 2.49 & 1.34 & 5.761 & 9.425 \\
\hline Big Lots Inc & 0.378 & 1.307 & 0.73 & 1.68 & 3.06 & 7.150 & 6.864 \\
\hline Dollar General & 0.151 & 0.137 & 0.48 & 1.72 & 1.37 & 3.849 & 3.562 \\
\hline Dollar Tree & 0.403 & 0.857 & 0.87 & 6.73 & 2.47 & 11.334 & 9.856 \\
\hline Family Dollar & 0.207 & 0.921 & 0.70 & 2.14 & 2.85 & 6.819 & 5.292 \\
\hline Fred's Inc & 0.568 & 0.686 & 0.26 & 1.93 & 3.09 & 6.534 & 6.686 \\
\hline Kohl's Corp & 0.232 & 0.881 & 0.47 & 1.11 & 1.24 & 3.932 & 4.485 \\
\hline Nordstrom, Inc & 0.474 & 0.165 & 0.49 & 1.18 & 1.30 & 3.615 & 6.180 \\
\hline JC Penny ${ }^{*}$ & 0.343 & 0.239 & 0.21 & 0.73 & 1.36 & 2.882 & 3.948 \\
\hline Dillard's, Inc & 0.239 & 0.849 & 0.20 & 0.59 & 1.43 & 3.312 & 3.382 \\
\hline PriceSmart, Inc & 0.136 & 0.059 & 0.45 & 4.44 & 2.58 & 7.663 & 4.448 \\
\hline Target Corp & 0.196 & 0.407 & 0.34 & 0.75 & 1.54 & 3.237 & 3.195 \\
\hline Whole Foods & 0.160 & 0.284 & 0.42 & 6.76 & 2.36 & 9.979 & 5.926 \\
\hline TJX Companies & 0.296 & 0.492 & 0.90 & 3.25 & 2.75 & 7.688 & 6.953 \\
\hline Coach Inc & 0.391 & -0.237 & 1.63 & 12.53 & 1.58 & 15.892 & 14.465 \\
\hline PetsMart & 0.267 & 0.854 & 0.57 & 2.81 & 2.31 & 6.807 & 5.779 \\
\hline Tiffany & 0.708 & 0.497 & 0.53 & 3.39 & 0.83 & 5.946 & 9.736 \\
\hline Ann & 0.255 & 0.905 & 0.54 & 1.68 & 2.49 & 5.870 & 5.007 \\
\hline Home Depot & 0.152 & 0.518 & 0.54 & 2.00 & 1.74 & 4.954 & 4.140 \\
\hline Tractor Supply & 0.473 & 1.007 & 0.73 & 6.41 & 2.65 & 11.278 & 9.922 \\
\hline Nike & 0.587 & 0.541 & 0.63 & 4.72 & 1.39 & 7.869 & 9.611 \\
\hline American & 0.542 & 1.271 & 0.39 & 3.58 & 1.62 & 7.400 & 8.377 \\
\hline
\end{tabular}

*J.C. Penney is a Solvent Firm but was predicted as a grey area firm according to the original Altman z-score 
Table 2. Bankrupt firms original Z-scores and BMW Z-scores

\begin{tabular}{lccccccc}
\hline Bankrupt & NWC/ TA & RE/TA & EBIT/TA & MVE/TL & Sales/TA & Z Score & BMW \\
\hline Blockbuster & 0.01 & -2.49 & 0.05 & $\$ 0.00$ & 2.0275 & $(0.40)$ & $(1.85)$ \\
Buffet Holdings & $(0.17)$ & -0.29 & 0.07 & $\$ 0.00$ & 1.6528 & 1.26 & $(1.76)$ \\
Borders Group & $(0.15)$ & -0.50 & -1.01 & $\$ 0.00$ & 2.3572 & 0.70 & $(5.28)$ \\
Lamonts & $(0.21)$ & -0.07 & -0.45 & $\$ 0.00$ & 2.2321 & 1.51 & $(3.71)$ \\
Ames & $(0.03)$ & -1.23 & -1.17 & $\$ 0.00$ & 3.0026 & 0.58 & $(5.43)$ \\
Linens n things & 0.29 & -0.28 & -0.36 & $\$ 0.00$ & 1.6058 & 1.25 & 0.84 \\
Shoe Pavilion & 0.31 & -0.09 & -0.64 & $\$ 0.00$ & 1.8968 & 1.47 & 0.22 \\
Tweeter & 0.18 & -1.30 & -0.17 & $\$ 0.00$ & 2.9983 & 1.70 & $(0.42)$ \\
Caldor & 0.06 & -0.71 & -0.46 & $\$ 0.00$ & 2.6306 & 1.52 & $(1.93)$ \\
Bradlees & $(0.07)$ & -0.03 & -0.07 & $\$ 0.03$ & 0.0032 & $(0.13)$ & $(0.84)$ \\
Merry Go Round & 0.25 & -0.28 & -0.87 & $\$ 0.00$ & 2.3836 & 1.48 & $(1.21)$ \\
Movie Gallery & $(0.03)$ & -0.54 & 0.28 & $\$ 0.00$ & 2.2041 & 1.91 & $(0.13)$ \\
Dairy Mart Stores & $(0.11)$ & -0.88 & -1.24 & $\$ 0.00$ & 3.9404 & 1.71 & $(6.27)$ \\
White Hall Jewelers & 0.31 & -0.78 & -1.08 & $\$ 0.00$ & 1.3143 & $(0.23)$ & $(1.53)$ \\
Shells & $(0.39)$ & -2.13 & -0.59 & $\$ 0.00$ & 3.4567 & 0.35 & $(7.34)$ \\
Eddie & 0.11 & -0.58 & -0.12 & $\$ 0.00$ & 1.2870 & 0.70 & $(0.08)$ \\
Samsonite & 0.27 & -1.60 & 0.40 & $\$ 0.18$ & 1.6439 & 0.90 & 2.34 \\
The Penntraffic & 0.32 & -0.67 & -0.38 & $\$ 0.00$ & 4.5030 & 3.77 & 0.32 \\
\hline "Movie Gallery is & & & & & & &
\end{tabular}

*Movie Gallery is in a grey area and went bankrupt and JC Penney is also in a grey area, but is not bankrupt according to original Altman z-score.

*The Penn traffic Company went bankrupt but the Original Altman z predicted Solvent.

*Samsonite is classified as a grey area with a score of 2.34 under BMW but if applied the cutoff of 2.675, it is correctly classified as bankrupt

Table 3. Solvent firms revised Z-scores

\begin{tabular}{llllll}
\hline Solvent & NWC/TA & RE/TA & EBIT/ TA & BVE/TL & Revised Z \\
\hline Aaron's, inc & 4.115067909 & 1.755885486 & 0.85 & 1.965487572 & 8.690042032 \\
Big Lots Inc & 2.065185185 & 3.042666667 & 1.48 & 1.477488856 & 8.066229597 \\
Dollar General & 0.826700189 & 0.317940499 & 0.97 & 0.918440691 & 3.030392504 \\
Dollar Tree & 2.204115918 & 1.996253675 & 1.78 & 1.661550976 & 7.639997007 \\
Family Dollar & 1.132016021 & 2.143591455 & 1.43 & 0.59787847 & 5.304513984 \\
Fred's Inc & 3.103892617 & 1.597181208 & 0.53 & 2.588372093 & 7.819378804 \\
Kohl's Corp & 1.269920834 & 2.051644902 & 0.95 & 1.189565594 & 5.462362130 \\
Nordstrom, Inc & 2.589010989 & 0.385328330 & 1.01 & 0.390011027 & 4.371179615 \\
JC Penny & 1.872633032 & 0.555414814 & 0.43 & 0.756132946 & 3.612875777 \\
Dillard's, Inc & 1.306300869 & 1.977315958 & 0.41 & 0.958176651 & 4.655071941 \\
PriceSmart, Inc & 0.740963855 & 0.137469880 & 0.92 & 1.370833333 & 3.170230924 \\
Target Corp & 1.072144606 & 0.947156618 & 0.69 & 0.576275781 & 3.286719895 \\
Whole Foods & 0.877315937 & 0.660810811 & 0.86 & 2.413950807 & 4.810083146 \\
TJX Companies & 1.618605118 & 1.145825389 & 1.82 & 0.668103448 & 5.256678461 \\
Coach Inc & 2.138535104 & -0.551787480 & 3.33 & 1.654545455 & 6.569414525 \\
PetsMart & 1.460728745 & 1.988995951 & 1.17 & 0.945000000 & 5.561882591 \\
Tiffany & 3.871734475 & 1.156183084 & 1.07 & 1.467843389 & 7.565996494 \\
Ann & 1.396216216 & 2.107252252 & 1.10 & 0.729389313 & 5.330155079 \\
Home Depot & 0.832830841 & 1.206468730 & 1.10 & 0.830809019 & 3.974850185 \\
Tractor Supply & 2.586984326 & 2.344338558 & 1.49 & 1.803066440 & 8.221636972 \\
Nike & 3.210017336 & 1.260918789 & 1.27 & 2.004878758 & 7.750096788 \\
American & 2.965617632 & 2.959231164 & 0.80 & 2.786235955 & 9.506738262 \\
\hline
\end{tabular}


Table 4. Bankrupt firms revised Z-scores

\begin{tabular}{lccccc}
\hline Bankrupt & NWC/TA & RE/TA & E BIT/TA & BVE/TL & Revised Z Score \\
\hline Blockbuster & 0.07 & -5.789434 & 0.096119 & 0.331336927 & $(5.29)$ \\
Buffet Holdings & $(0.94)$ & -0.681661 & 0.143732 & -0.18158433 & $(1.66)$ \\
Borders Group & $(0.80)$ & -1.156731 & -2.06469 & -0.14429989 & $(4.17)$ \\
Lamonts & $(1.16)$ & -0.154884 & -0.91129 & 0.19336007 & $(2.04)$ \\
AmesDepartment & & & & & \\
Stores Inc & $(0.19)$ & -2.853277 & -2.37586 & -0.29112877 & 0.239081887 \\
Linens n things & 1.56 & -0.653024 & -0.73877 & 0.474601971 & 0.41 \\
Shoe Pavilion & 1.69 & -0.219865 & -1.30961 & & 0.64 \\
TweeterHome & & & & 0.386518628 & $(2.00)$ \\
Entertainment & 1.00 & -3.030963 & -0.35534 & -0.24095694 & $(2.52)$ \\
Caldor & 0.31 & -1.653559 & -0.93324 & 0.114365862 & $(0.48)$ \\
Bradlees & $(0.39)$ & -0.064527 & -0.14187 & 0.025092673 & $(1.02)$ \\
Merry Go Round & 1.39 & -0.658128 & -1.7746 & -0.17863648 & $(1.03)$ \\
Movie Gallery & $(0.16)$ & -1.253942 & 0.561448 & -0.35943358 & $(5.55)$ \\
Dairy Mart Stores & $(0.62)$ & -2.044147 & -2.52652 & & $(2.12)$ \\
WhiteHall & & & & 0.183172041 & $(7.77)$ \\
Jewelers & 1.72 & -1.813038 & -2.20202 & 0.507542145 & $(0.52)$ \\
Shells & $(2.11)$ & -4.97145 & -1.19819 & -0.484699186 & $(1.67)$ \\
Eddie Bauer & 0.58 & -1.353196 & 0.823997 & -0.27445236 & \\
Samsonite & 1.50 & -3.718545 & & 0.240386165 & $(0.33)$ \\
ThePenntraffic & & & -0.78282 & \\
Company & 1.76 & -1.551678 & &
\end{tabular}

Table 5. Descriptive statistics of revised and original Z-scores

\begin{tabular}{|c|c|c|c|c|c|c|}
\hline & Solvent & Rev & Solvent & Bank & Bankrupt & Solvent \\
\hline Statistic & Rev & Bankrupt & Orig & Orignial & BMW & BMW \\
\hline MEAN & 5.893478487 & $(2.38)$ & 6.807702183 & 1.11 & (1.89) & 6.69 \\
\hline MEDIAN & 5.396258604 & $(2.00)$ & 6.670545013 & 1.25 & $(1.37)$ & 6.05 \\
\hline $\mathrm{MIN}$ & 3.030392504 & (7.77) & 2.88196739 & $(0.40)$ & $(7.34)$ & 3.19 \\
\hline MAX & 9.506738262 & 0.64 & 15.89198896 & 3.77 & 2.34 & 14.47 \\
\hline
\end{tabular}

The original Altman z-score predicted Wal-Mart correctly, but the revised Altman z-score labeled the company in the grey area category. Sears Holdings Inc, which is a solvent retail firm, was predicted as a bankrupt firm according to the revised Altman $\mathrm{z}$ function and as indecisive for the original Altman $z$ function. Sears was misclassified in all z-score tests because it is still a functioning company, but has been operating with losses and with high leverage. Figure 2 shows Sears Holding Corp were classified as a bankrupt firm because of its poor ratios.

Lastly, Circuit City, which is a bankrupt retail company, was categorized as solvent according to the original Altman model with a score of 3.47 and a score of 2.4 for the revised Altman model. Clearly, all models are not a hundred percent accurate. Table 6 presents all of the results. The highlighted scores in Table 6 are the wrong predictions.

\section{MULTIPLE DISCRIMINANT MODELS}

\subsection{Formulation of Multiple Discriminant Model}

As mentioned in the introduction of this study, the main goal is to formulate better multiple discriminant functions that will predict bankruptcy more accurately for retail firms than Begley, Ming and Watts and Altman's models. Here will describe the process of making better discriminant models. 


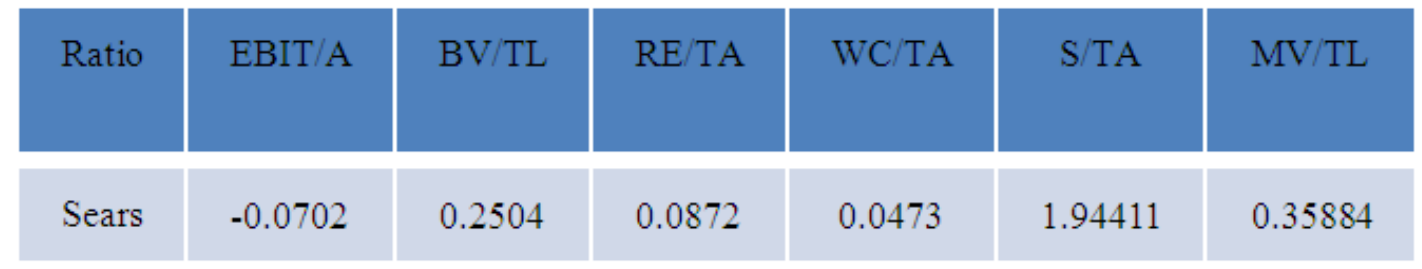

Fig. 2. Misclassification from the Revised Altman Z Model

Table 6. Test retail companies

\begin{tabular}{llcrr}
\hline Test Company & Group & BMW & Original Z & Revised Altman Z \\
\hline WMT & Solvent & 1.52398711 & 4.22 & 2.445477525 \\
URBAN & Solvent & 8.01383573 & 9.58 & 7.954426497 \\
SAKS & Solvent & 4.38835506 & 3.10 & 3.886651016 \\
Stein Mart & Solvent & 4.93495998 & 4.56 & 5.739780247 \\
Fossil & Solvent & 13.46875270 & 13.31 & 10.211005620 \\
GNC & Solvent & 3.88464381 & 3.08 & 3.116483606 \\
Vitamin & Solvent & 5.86933536 & 8.42 & 5.395577796 \\
ANF & Solvent & 4.87568694 & 4.97 & 6.237163396 \\
ARO & Solvent & 6.82284905 & 8.12 & 6.617821084 \\
Children & Solvent & 7.12227099 & 6.51 & 7.625948969 \\
Sears & Solvent & -0.38784670 & 2.11 & 0.385736000 \\
Costco & Solvent & 2.07281780 & 5.59 & 2.738954000 \\
Liz Claiborne & Solvent & 4.97471350 & 5.03 & 6.417403000 \\
Circuit City & Bankrupt & 1.06304090 & 3.47 & 2.396020000 \\
Finlay Enterprises & Bankrupt & 0.71536000 & 0.91 & 0.314585000 \\
\hline
\end{tabular}

All bankrupt firms were labeled as zeros and solvent firms were labeled as ones to distinguish between the two groups. The zeros and ones are classified as the dependent variable and were regressed against the four financial ratio variables used in the revised Altman $\mathrm{z}$ model. These ratios are Net Working Capital to Total Assets (NWC/TA), Retained Earnings to Total Assets (RE/TA), Earnings Before Interest and Taxes to Total Assets (EBIT/TA) and Book Value of Equity to Total Liabilities (BVE/TL). Collectively, these four ratios predict bankruptcy more accurately than the original five financial ratios used in Altman's original model. Market Value of Equity to Total Liabilities (MVE/TL) and sales to total assets were not included in the new discriminant model because they predict bankruptcy more accurately for manufacturing firms rather than retail. The same companies and financial data used to test the Altman and BMW models were used to formulate the model. As mentioned before, data for the bankrupt retailers was collected a year before each went bankrupt. Bankrupt data was sampled from EDGAR Company Search and the most recent data of solvent firms was retrieved from Charles Schwab. Excel was used to compute the regression formula and the regression output is listed below, labeled Table 7:

$$
\begin{aligned}
& \text { Bankruptcy Score }=0.392 \\
& +1.02 \frac{\mathrm{EBIT}}{\mathrm{TA}}+0.159 \frac{\mathrm{BVE}}{\mathrm{TL}} \\
& +0.312 \frac{\mathrm{RE}}{\mathrm{TA}}+0.0511 \frac{\mathrm{NWC}}{\mathrm{TA}}
\end{aligned}
$$

The above equation from the regression will predict whether the firm is labeled as a bankrupt firm (zero) or a solvent firm (one). One can see that the last explanatory variable, NWC/TA is not significant because its $\mathrm{P}$-value is much greater. However, NWC/TA was still included in the discriminant function for now because NWC/TA was also included in Altman's revised z-score model. Notice that NWC/TA will be removed later in a revised model as a comparison. 
Table 7. Regression output

\begin{tabular}{|c|c|c|c|c|c|c|}
\hline \multicolumn{7}{|l|}{ Regression Statistics } \\
\hline Multiple R & 0.91195 & & & & & \\
\hline R Square & 0.831652 & & & & & \\
\hline Adjusted R Square & 0.812413 & & & & & \\
\hline Standard Error & 0.218216 & & & & & \\
\hline Observation & 40 & & & & & \\
\hline ANOVA & $\mathrm{df}$ & SS & MS & $\mathrm{F}$ & Significance F & \\
\hline Regression & 4 & 8.233357 & 2.05833932 & 43.22575 & 4.47E-13 & \\
\hline Residual & 35 & 1.666643 & 0.04761836 & & & \\
\hline \multirow{2}{*}{ Total } & 39 & 9.9 & & & & \\
\hline & Coefficients & Standard Error & t Stat & P-value & Lower 95\% & Upper $95 \%$ \\
\hline Intercept & 0.391562 & 0.060186 & 6.50583066 & $1.67 \mathrm{E}-07$ & 0.269377 & 0.513747 \\
\hline EBIT/TA & 1.026273 & 0.238999 & 4.29404946 & 0.000132 & 0.54108 & 1.511467 \\
\hline $\mathrm{BV} / \mathrm{TL}$ & 0.159101 & 0.067546 & 2.35545527 & 0.024233 & 0.021976 & 0.296226 \\
\hline RE/TA & 0.31209 & 0.075671 & 4.12429102 & 0.000218 & 0.158469 & 0.465711 \\
\hline NWC/TA & 0.05107 & 0.234068 & 0.21818657 & 0.828551 & -0.42411 & 0.526253 \\
\hline
\end{tabular}

Ratios that are weak and undesirable will compute a score close to 0 , while strong ratios will compute a score close to 1. Table 8 shows discriminant scores and ratios for all forty firms. The discriminant score is just the predicted value from the estimated regression equation. These discriminant scores are used to classify each of the observations in the sample. A cutoff value $\mathrm{C}$ is used to determine where the company falls under, bankrupt or solvent. The cutoff value in this example was 0.55 and is calculated as followed Equation 5:

$\mathrm{C}=\frac{\mathrm{n}_{0} \overline{\mathrm{d}}_{0}+\mathrm{n}_{1} \overline{\mathrm{d}}_{1}}{\text { Sample size }}$

Where:

$\overline{\mathrm{d}_{0}}=$ Average score for bankrupt firms

$\overline{\mathrm{d}_{1}}=$ Average score for solvent firms

$\mathrm{n}_{0}=$ Total \# of bankrupt firms

$\mathrm{n}_{1}=$ Total \# of solvent firms

If $\mathrm{d} \leq \mathrm{c}$, assign to group 0

If $\mathrm{d} \geq \mathrm{c}$, assign to group 1

All of the observations according to the model and cut off value predicted correctly. There were no misclassifications as observed above. Even though in this case regression was used to predict bankruptcy, this is not the method of choice. A more appropriate method would be actual discriminant analysis. Discriminant analysis is used more often than regression to predict groups because it can be extended to more than two groups and it also computes the means of each of the different groups for each independent variable which measures of the distance from each data to each set of averages. Each data is classified into the appropriate group whose set of averages is the closest. MINITAB was used to compute two linear discriminant functions for the two groups. The discriminant functions for the two groups are:

$$
\begin{gathered}
\mathrm{B}_{\mathrm{s}}=\beta_{\mathrm{B} 0}+\beta_{\mathrm{B} 1} \mathrm{X}_{\mathrm{B} 1}+\beta_{\mathrm{B} 2} \mathrm{X}_{\mathrm{B} 2}+\beta_{\mathrm{B} 3} \mathrm{X}_{\mathrm{B} 3}+\beta_{\mathrm{B} 4} \mathrm{X}_{\mathrm{B} 4} \\
\mathrm{~S}_{\mathrm{s}}=\beta_{\mathrm{S} 0}+\beta_{\mathrm{S} 1} \mathrm{X}_{\mathrm{S} 1}+\beta_{\mathrm{S} 2} \mathrm{X}_{\mathrm{S} 2}+\beta_{\mathrm{S} 3} \mathrm{X}_{\mathrm{S} 3}+\beta_{\mathrm{S} 4} \mathrm{X}_{\mathrm{S} 4} \\
\mathrm{~B}_{\mathrm{s}}=\text { Bankrupt Score and } \mathrm{S}_{\mathrm{s}}=\text { Solvent Score. }
\end{gathered}
$$

$$
\begin{gathered}
\text { If } \mathrm{B}_{\mathrm{s}}>\mathrm{S}_{\mathrm{s}}, \text { firm }=\text { bankrupt. } \\
\text { If } \mathrm{S}_{\mathrm{s}}>\mathrm{B}_{\mathrm{s}} \text {, firm }=\text { Solvent } \\
\mathrm{X}_{1}=\frac{\text { EBIT }}{\mathrm{TA}} \text { or Earnings before interest }
\end{gathered}
$$$$
\text { and taxes to total assets }
$$

$$
\mathrm{X}_{2}=\frac{\mathrm{BVE}}{\mathrm{TL}} \text { or Book Value of }
$$

equity to total liabilities

$$
\mathrm{X}_{3}=\frac{\text { R.E. }}{\mathrm{TA}} \text { or Retained earnings }
$$

to total assets

$$
\begin{aligned}
& \mathrm{X}_{4}=\frac{\mathrm{NWC}}{\mathrm{TA}} \text { or Net Working Capital } \\
& \text { to total assets }
\end{aligned}
$$


Table 8. Discriminant scores of the forty firms

\begin{tabular}{|c|c|c|c|c|c|c|}
\hline Company & Group & EBIT/TA & $\mathrm{BV} / \mathrm{TL}$ & RE/TA & NWC/TA & Score \\
\hline Blockbuster & Bankrupt & 0.014303 & 0.315559 & -1.77590 & 0.01134 & -0.097213974 \\
\hline Buffet Holdings & Bankrupt & 0.021389 & -0.172940 & -0.20910 & -0.14358 & 0.313408279 \\
\hline Borders Group & Bankrupt & -0.307250 & -0.137430 & -0.35483 & -0.12201 & -0.062589255 \\
\hline Lamonts & Bankrupt & -0.135610 & 0.184152 & -0.04751 & -0.17745 & 0.257799129 \\
\hline \multicolumn{7}{|l|}{ Ames Department } \\
\hline Stores Inc & Bankrupt & -0.353550 & -0.277270 & -0.87524 & -0.028770 & -0.290013263 \\
\hline Linens $n$ things & Bankrupt & -0.109940 & 0.227697 & -0.20031 & 0.238102 & 0.264608835 \\
\hline Shoe Pavilion & Bankrupt & -0.194880 & 0.452002 & -0.06744 & 0.257751 & 0.255588158 \\
\hline \multicolumn{7}{|l|}{ Tweeter Home } \\
\hline \multicolumn{7}{|l|}{ Entertainment } \\
\hline Group & Bankrupt & -0.052880 & 0.368113 & -0.92974 & 0.152200 & 0.113472382 \\
\hline Caldor & Bankrupt & -0.138870 & -0.229480 & -0.50723 & 0.047399 & 0.056647958 \\
\hline Bradlees & Bankrupt & -0.021110 & 0.108920 & -0.01979 & -0.059200 & 0.378024394 \\
\hline Merry Go Round & Bankrupt & -0.264080 & 0.023898 & -0.20188 & 0.211194 & 0.072130226 \\
\hline Movie Gallery & Bankrupt & 0.083549 & -0.170130 & -0.38464 & -0.02498 & 0.328919002 \\
\hline \multicolumn{7}{|l|}{ Dairy Mart } \\
\hline Stores & Bankrupt & -0.375970 & -0.342320 & -0.62704 & -0.09471 & -0.249278472 \\
\hline \multicolumn{7}{|l|}{ White Hall } \\
\hline Jewelers & Bankrupt & -0.327680 & 0.174450 & -0.55615 & 0.261550 & -0.077183396 \\
\hline Shells & Bankrupt & -0.178300 & 0.483373 & -1.52498 & -0.321300 & -0.206860909 \\
\hline Eddie & Bankrupt & -0.034970 & 0.461618 & -0.41509 & 0.088511 & 0.304093111 \\
\hline Samsonite & Bankrupt & 0.122619 & -0.261380 & -1.14066 & 0.228266 & 0.131485815 \\
\hline \multicolumn{7}{|l|}{ The Penntraffic } \\
\hline Company & Bankrupt & -0.116490 & 0.228939 & -0.475970 & 0.268587 & 0.173604693 \\
\hline Aaron's, inc & Solvent & 0.127024 & 1.871893 & 0.538615 & 0.627297 & 1.019876578 \\
\hline Big Lots Inc & Solvent & 0.220370 & 1.407132 & 0.933333 & 0.314815 & 1.148960509 \\
\hline Dollar General & Solvent & 0.143945 & 0.874705 & 0.097528 & 0.126021 & 0.715329322 \\
\hline Dollar Tree & Solvent & 0.264595 & 1.58243 & 0.612348 & 0.335993 & 1.123141987 \\
\hline Family Dollar & Solvent & 0.212951 & 0.569408 & 0.657543 & 0.172563 & 0.914726824 \\
\hline Fred's Inc & Solvent & 0.078859 & 2.465116 & 0.489933 & 0.473154 & 1.041763345 \\
\hline Kohl’s Corp & Solvent & 0.141552 & 1.132920 & 0.629339 & 0.193585 & 0.923379157 \\
\hline Nordstrom, Inc & Solvent & 0.149826 & 0.371439 & 0.118199 & 0.394666 & 0.661465317 \\
\hline JC Penny & Solvent & 0.063794 & 0.720127 & 0.170373 & 0.285462 & 0.639355483 \\
\hline Dillard's, Inc & Solvent & 0.061500 & 0.912549 & 0.606539 & 0.199131 & 0.799329780 \\
\hline PriceSmart, Inc & Solvent & 0.137048 & 1.305556 & 0.042169 & 0.112952 & 0.758855467 \\
\hline Target Corp & Solvent & 0.102849 & 0.548834 & 0.290539 & 0.163437 & 0.683454247 \\
\hline Whole Foods & Solvent & 0.127679 & 2.299001 & 0.202703 & 0.133737 & 0.958461363 \\
\hline TJX Companies & Solvent & 0.271450 & 0.636289 & 0.351480 & 0.246739 & 0.893673019 \\
\hline Coach Inc & Solvent & 0.495256 & 1.575758 & -0.169260 & 0.325996 & 1.114359728 \\
\hline PetsMart & Solvent & 0.173684 & 0.900000 & 0.610121 & 0.222672 & 0.914785490 \\
\hline Tiffany & Solvent & 0.159261 & 1.397946 & 0.354657 & 0.590203 & 0.918249595 \\
\hline Ann & Solvent & 0.163288 & 0.694656 & 0.646396 & 0.212838 & 0.882264851 \\
\hline Home Depot & Solvent & 0.164396 & 0.791247 & 0.370082 & 0.126956 & 0.808148516 \\
\hline Tractor Supply & Solvent & 0.221317 & 1.717206 & 0.719122 & 0.394357 & 1.136473876 \\
\hline Nike & Solvent & 0.189625 & 1.909408 & 0.386785 & 0.489332 & 1.035660749 \\
\hline American & Solvent & 0.118401 & 2.653558 & 0.907740 & 0.452076 & 1.241642085 \\
\hline
\end{tabular}


The MINITAB output is listed below and the results show that there were no misclassifications of the predicted data:

Discriminant Analysis: Bankruptcy versus EBIT/TA, BVE/TL, RE/TA, NWC/TA

Linear Method for Response: Bankruptcy Predictors: EBIT/TA, BVE/TL, RE/TA, NWC/TA

$\begin{array}{lll}\text { Group } & 0 & 1 \\ \text { Count } & 18 & 22\end{array}$

Summary of classification

$\begin{array}{lll} & \text { True } & \text { Group } \\ \text { Put into Group } & 0 & 1 \\ 0 & 18 & 0 \\ 1 & 0 & 22 \\ \text { Total N } & 18 & 22 \\ \text { N correct } & 18 & 22 \\ \text { Proportion } & 1.000 & 1.000 \\ \mathrm{~N}=40 & \text { N Correct }=40\end{array}$

Proportion Correct $=1.000$

Squared Distance Between Groups

$\begin{array}{lll} & 0 & 1 \\ 0 & 0.0000 & 18.9619 \\ 1 & 18.9619 & 0.0000\end{array}$

Linear Discriminant Function for Groups

$\begin{array}{lll} & 0 & 1 \\ \text { Constant } & -2.138 & -4.802 \\ \text { EBIT/TA } & -11.465 & 11.935 \\ \text { BVE/TL } & 0.220 & 3.848 \\ \text { RE/TA } & -4.512 & 2.604 \\ \text { NWC/TA } & 3.707 & 4.871\end{array}$

The new Discriminant functions for bankrupt and solvent firms is:

$$
\begin{aligned}
\text { Bankrupt Score } & =-2.138-11.465 \frac{\mathrm{EBIT}}{\mathrm{TA}} \\
& +0.22 \frac{\mathrm{BVE}}{\mathrm{TL}}-4.512 \frac{\mathrm{RE}}{\mathrm{TA}} \\
& +3.707 \frac{\mathrm{NWC}}{\mathrm{TA}}
\end{aligned}
$$

$$
\begin{aligned}
\text { Solvent Score } & =-4.802+11.935 \frac{\mathrm{EBIT}}{\mathrm{TA}} \\
& +3.848 \frac{\mathrm{BVE}}{\mathrm{TL}}+2.604 \frac{\mathrm{RE}}{\mathrm{TA}} \\
& +4.871 \frac{\mathrm{NWC}}{\mathrm{TA}}
\end{aligned}
$$

These equations are applied to each retail company and then the retail company is then classified into the group for which it's score is the highest. For instance, if its solvent score is higher than its bankrupt score, then that retail firm is classified as solvent. Equation 6 and 7 do not produce z-scores like Altman's model. These are much simpler models because they label a retail firm as bankrupt or solvent and don't produce grey area values like Altman's models. The chart listed below shows each of the forty firm's bankrupt and solvent discriminant scores and its prediction from those scores, whether it went bankrupt (zero) or solvent (one). As one can see in Table 9 below, the discriminant functions accurately predicted bankruptcy. The first eighteen retail firms went bankrupt (labeled bankrupt) and the next twenty two were solvent (labeled solvent). The first eighteen retail firms that went bankrupt had bankrupt scores greater than their solvent score. Hence, they were labeled with a zero. The twenty two solvent retail companies had solvent scores that were greater than their bankrupt scores and were given a prediction of one. This can be seen in the prediction column below. The prediction column was calculated in Excel as followed Equation 8:

$$
\begin{aligned}
& \text { Prediction of } 0 \text { or } 1 \\
& =\text { if }(\text { Bankrupt Score }>\text { Solvent, } 0,1)
\end{aligned}
$$

This Excel function means that if the bankruptcy discriminant score was greater than the solvent score than the observation was given a zero. However if it was not true then it gave the observation a one.

After the scores were tested out on the original forty companies, a second test was implemented on the second set of fifteen retail firms from before and was compared to their original and revised Altman z-scores. In this data set the original $z$-score is $86.67 \%$ accurate. Sears and Circuit City were predicted wrong because the original Altman z-score predicted Sears as inconclusive (and is solvent) and predicted Circuit City as solvent and is bankrupt. The revised Altman z-score and the BMW model were even worse because they were only $80 \%$ correct. However, the new discriminant functions were $93.33 \%$ correct. It only predicted Sears as insolvent. Table 10 shows the results. 
Table 9. Predictions of the forty firms

\begin{tabular}{|c|c|c|c|c|}
\hline Company & Group & Bankrupt Score & Solvent Score & Prediction \\
\hline Blockbuster & Bankrupt & 5.822332655 & -7.986221481 & 0 \\
\hline Buffet Holdings & Bankrupt & -2.010057702 & -6.456048273 & 0 \\
\hline Borders Group & Bankrupt & 2.503031104 & -10.516063580 & 0 \\
\hline Lamonts & Bankrupt & -0.986182952 & -6.699967474 & 0 \\
\hline \multicolumn{5}{|l|}{ Ames Department } \\
\hline Stores Inc & Bankrupt & 5.696880199 & -12.507815580 & 0 \\
\hline Linens $n$ things & Bankrupt & 0.958970354 & -4.599728642 & 0 \\
\hline Shoe Pavilion & Bankrupt & 1.455562234 & -4.308741146 & 0 \\
\hline \multicolumn{5}{|l|}{ Tweeter Home } \\
\hline \multicolumn{5}{|l|}{ Entertainment } \\
\hline Group & Bankrupt & 3.308431524 & -5.696274145 & 0 \\
\hline Caldor & Bankrupt & 1.868028789 & -8.432461974 & 0 \\
\hline Bradlees & Bankrupt & -2.002123131 & -4.974736250 & 0 \\
\hline Merry Go Round & Bankrupt & 2.588680299 & -7.358770535 & 0 \\
\hline Movie Gallery & Bankrupt & -1.490390824 & -5.582784873 & 0 \\
\hline Dairy Mart Stores & Bankrupt & 4.575286343 & -12.700596510 & 0 \\
\hline White Hall Jewelers & Bankrupt & 5.136142897 & -8.215790018 & 0 \\
\hline Shells & Bankrupt & 5.702273746 & -10.606111280 & 0 \\
\hline Eddie & Bankrupt & 0.565477854 & -4.092805852 & 0 \\
\hline Samsonite & Bankrupt & 2.391507164 & -6.202737956 & 0 \\
\hline \multicolumn{5}{|l|}{ The Penntraffic } \\
\hline Company & Bankrupt & 2.391189162 & -5.242518773 & 0 \\
\hline Aaron's, inc & Solvent & -3.287355294 & 8.375192346 & 1 \\
\hline Big Lots Inc & Solvent & -7.399158684 & 7.206628207 & 1 \\
\hline Dollar General & Solvent & -3.568779505 & 1.149663702 & 1 \\
\hline Dollar Tree & Solvent & -6.340829810 & 7.676303378 & 1 \\
\hline Family Dollar & Solvent & -6.781352054 & 2.483447062 & 1 \\
\hline Fred's Inc & Solvent & -2.956387506 & 9.205470462 & 1 \\
\hline Kohl's Corp & Solvent & -5.633609502 & 3.828653709 & 1 \\
\hline Nordstrom, Inc & Solvent & -2.844321329 & 0.645677749 & 1 \\
\hline JC Penny & Solvent & -2.421481591 & 0.564564852 & 1 \\
\hline Dillard's, Inc & Solvent & -4.640856919 & 1.992883892 & 1 \\
\hline PriceSmart, Inc & Solvent & -3.193588019 & 2.517443440 & 1 \\
\hline Target Corp & Solvent & -3.901467692 & 0.090075297 & 1 \\
\hline Whole Foods & Solvent & -3.514895041 & 6.747680307 & 1 \\
\hline TJX Companies & Solvent & -5.781410173 & 3.003314752 & 1 \\
\hline Coach Inc & Solvent & -5.497276407 & 8.319572078 & 1 \\
\hline PetsMart & Solvent & -5.858712146 & 3.407512955 & 1 \\
\hline Tiffany & Solvent & -3.068712033 & 6.276488183 & 1 \\
\hline Ann & Solvent & -5.984826473 & 2.539833213 & 1 \\
\hline Home Depot & Solvent & -5.047913019 & 1.786881292 & 1 \\
\hline Tractor Supply & Solvent & -6.080406500 & 8.240732083 & 1 \\
\hline Nike & Solvent & -3.823204135 & 8.199304685 & 1 \\
\hline American & Solvent & -5.331558592 & 11.387820650 & 1 \\
\hline
\end{tabular}


Table 10. Comparisons of the discriminant Model to BMW, altman $\mathrm{Z}$ and revised altman $\mathrm{Z}$ models

\begin{tabular}{|c|c|c|c|c|c|c|}
\hline Test Company & Group & $\begin{array}{l}\text { Bankrupt } \\
\text { Score }\end{array}$ & $\begin{array}{l}\text { Solvent } \\
\text { Score }\end{array}$ & BMW & $\begin{array}{l}\text { Original } \\
\mathrm{Z}\end{array}$ & $\begin{array}{l}\text { Revised } \\
\text { Altman Z }\end{array}$ \\
\hline WMT & Solvent & $(5.33)$ & $(0.18)$ & 1.524 & 4.216 & 2.445 \\
\hline URBAN & Solvent & $(6.15)$ & 10.42 & 8.014 & 9.581 & 7.954 \\
\hline SAKS & Solvent & (1.34) & 2.58 & 4.388 & 3.098 & 3.887 \\
\hline Stein Mart & Solvent & $(3.63)$ & 3.67 & 4.935 & 4.556 & 5.740 \\
\hline Fossil & Solvent & $(6.88)$ & 11.25 & 13.469 & 13.315 & 10.211 \\
\hline GNC & Solvent & $(3.06)$ & 0.36 & 3.885 & 3.078 & 3.116 \\
\hline Vitamin & Solvent & $(3.72)$ & 8.42 & 5.869 & 8.415 & 5.396 \\
\hline ANF & Solvent & (4.99) & 5.22 & 4.876 & 4.965 & 6.237 \\
\hline $\mathrm{ARO}$ & Solvent & $(5.20)$ & 5.15 & 6.823 & 8.124 & 6.618 \\
\hline Children & Solvent & $(3.61)$ & 9.61 & 7.122 & 6.508 & 7.626 \\
\hline Sears & Solvent & $(1.50)$ & $(4.22)$ & -0.388 & 2.107 & 0.386 \\
\hline $\begin{array}{l}\text { Costco } \\
\text { Liz }\end{array}$ & Solvent & $(3.97)$ & 0.41 & 2.073 & 5.593 & 2.739 \\
\hline $\begin{array}{l}\text { Claiborne } \\
\text { Circuit }\end{array}$ & Solvent & $(9.96)$ & 1.32 & 4.975 & 5.033 & 6.417 \\
\hline $\begin{array}{l}\text { City } \\
\text { Finlay }\end{array}$ & Bankrupt & $(1.33)$ & $(1.54)$ & 1.063 & 3.469 & 2.396 \\
\hline Enterprises & Bankrupt & 1.60 & $(5.86)$ & 0.715 & 0.908 & 0.315 \\
\hline
\end{tabular}

The discriminant function misclassified Sears Holding Company as bankrupt. This could be from the NWC/TA explanatory variable. Recall from before when the regression was implemented. The regression said that it had a high p-value of $82 \%$. NWC/TA should not be included in the regression and in the discriminant models. If one looks at the solvent score and bankruptcy Score models, he can see that the weights of the NWC/TA coefficients are very similar. The coefficient weight for NWC/TA in the solvent and bankruptcy Score models is 4.871 and 3.707 respectively. Since they are not that different, NWC/TA should be removed.

The same process was used to come up with two new discriminant functions without NWC/TA. Two groups were created, bankrupt (zero) and solvent (one) for each of the original forty retail firms. Results from MINITAB are displayed below:

Discriminant Analysis: Bankruptcy versus RE/TA, BV/TL, EBIT/TA

Linear Method for Response: Bankruptcy

Predictors: RE/TA, BV/TL, EBIT/TA

$\begin{array}{lll}\text { Group } & 0 & 1 \\ \text { Count } & 18 & 22\end{array}$

Summary of classification

True Group

$\begin{array}{lll}\text { Put into Group } & 0 & 1 \\ 0 & 18 & 0 \\ 1 & 0 & 22\end{array}$

$\begin{array}{lll}\text { Total N } & 18 & 22 \\ \text { N correct } & 18 & 22 \\ \text { Proportion } & 1.000 & 1.000 \\ & \\ \mathrm{~N}=40 \mathrm{~N} & \text { Correct }=40 \\ \text { Proportion Correct }=1.000\end{array}$

Squared Distance Between Groups

$\begin{array}{lll} & 0 & 1 \\ 0 & 0.0000 & 18.9310 \\ 1 & 18.9310 & 0.0000\end{array}$

Linear Discriminant Function for Groups

$\begin{array}{lll} & 0 & 1 \\ \text { Constant } & -1.981 & -4.531 \\ \text { RE/TA } & -4.260 & 2.934 \\ \text { BV/TL } & 0.637 & 4.396 \\ \text { EBIT/TA } & -11.162 & 12.332\end{array}$

The new Linear Discriminant Functions are:

$$
\begin{aligned}
\text { Bankrupt Score } & =-1.981-4.26 \frac{\mathrm{RE}}{\mathrm{TA}}+0.637 \frac{\mathrm{BVE}}{\mathrm{TL}} \\
& -11.162 \frac{\mathrm{EBIT}}{\mathrm{TA}}
\end{aligned}
$$

Solvent Score $=-4.531+2.934 \frac{\mathrm{RE}}{\mathrm{TA}}+4.396 \frac{\mathrm{BVE}}{\mathrm{TL}}$

$$
-12.332 \frac{\mathrm{EBIT}}{\mathrm{TA}}
$$


Equation 9 and 10 were tested using both the original forty retail firms and second data set of fifteen retail firms. MINITAB output from above shows that all of the forty firms were correctly predicted. The prediction outcomes of the second data set are presented in Table 11.

It was hypothesized that these two new models excluding NWC/TA would better predict bankruptcy and solvency better than the original models with NWC/TA variable. However, this is not the case because it misclassified more retail firms than the original. In this case, it misclassified Sears Holdings Company and Circuit City as wrong predictions. These functions are only $86.67 \%$ accurate, while the original is $93 \%$ accurate. Hence, NWC/TA should be included in the two original discriminant functions because it does make a difference between the two groups and is important explanatory variable despite it having a low $\mathrm{T}$ Statistic and a high P-value.

\subsection{Formulation of Other Multiple Discriminant Models}

Instead of using the variables in Altman's revised discriminant equation, one can use other financial ratios to determine bankruptcy. Here, we will provide two extra discriminant functions that use different financial ratios as explanatory variables. The first discriminant function will use the debt ratio, the current ratio and the return on assets ratio. The last discriminant function will use only the current ratio and the profit margin ratio:

Debt Ratio $=$ Long Term + Short Term Debt $/$ Total Assets. The debt ratio is a leverage ratio that tells the analyst the percentage of assets that are supported by the retail firm's total debt.

Current Ratio $=$ Current Assets/Current Liabilities. This is a liquidity ratio that tells the analyst if the company can meet its current obligations or not. If the company can't then it could go bankrupt.

Return on Assets $=$ Net Income/Total Assets. The ROA equation tells the analyst how much return does the firm have for every dollar in assets. This is a popular profitability ratio. A negative ROA implies that a firm is not profitable and could go bankrupt. It would be wise to use the Return on Assets formula instead of Return on Equity ratio when predicting bankruptcy. If the company has negative equity and negative income then mathematically those values make the ratio positive. Return on Assets on the other hand will not do this because it is impossible for a firm to have negative assets.

Both bankrupt and solvent groups were classified as zeros and ones respectively. The MINITAB discriminant functions produced two models for the two groups. The output is below.

Discriminant Analysis: Bankruptcy versus ROA, Debt Ratio, Current Ratio

Linear Method for Response: Bankruptcy

Predictors: ROA, Debt Ratio, Current Ratio

$\begin{array}{lll}\text { Group } & 0 & 1 \\ \text { Count } & 18 & 22\end{array}$

Count $\quad 18 \quad 22$

Table 11. Predictions of the fifteen firms without NWC/TA

\begin{tabular}{lllrl}
\hline & & Bankrupt & \multicolumn{1}{l}{ Solvent } \\
Test Company & Group & Score & Score & Prediction \\
\hline WMT & Solvent & -6.158240938 & 2.819544115 & 1 \\
URBAN & Solvent & -9.277446308 & 16.238899470 & 1 \\
SAKS & Solvent & -1.176555276 & 1.127588069 & 1 \\
Stein Mart & Solvent & -7.247025241 & 7.232520251 & 1 \\
Fossil & Solvent & -11.302084180 & 15.761344910 & 1 \\
GNC & Solvent & -3.385704582 & 0.269954792 & 1 \\
Vitamin & Solvent & -3.205528044 & 10.007881980 & 1 \\
ANF & Solvent & -9.746144495 & 11.944147180 & 1 \\
ARO & Solvent & -8.813108814 & 9.140504545 & 1 \\
Children & Solvent & -5.955994797 & 12.539668670 & 1 \\
Sears & Solvent & -2.495769600 & -2.560858000 & 0 \\
Costco & Solvent & -4.816821000 & 2.587727000 & 1 \\
Liz Claiborne & Solvent & -17.565464500 & 11.796587000 & 1 \\
Circuit City & Bankrupt & -4.095254000 & 1.381244000 & 1 \\
Finlay Enterprises & Bankrupt & 0.042728500 & -6.370183200 & 0 \\
\hline
\end{tabular}


Summary of classification

$\begin{array}{lll} & \text { True } & \text { Group } \\ \text { Put into Group } & 0 & 1 \\ 0 & 18 & 0 \\ 1 & 0 & 22 \\ \text { Total N } & 18 & 22 \\ \text { N correct } & 18 & 22 \\ \text { Proportion } & 1.000 & 1.000\end{array}$

$\mathrm{N}=40 \quad \mathrm{~N}$ Correct $=40$

Proportion Correct $=1.000$

Squared Distance Between Groups

$\begin{array}{lll} & 0 & 1 \\ 1 & 0.0000 & 21.5553 \\ & 21.5553 & 0.0000\end{array}$

Linear Discriminant Function for Groups

$\begin{array}{lll} & 0 & 1 \\ \text { Constant } & -11.852 & -5.722 \\ \text { ROA } & -9.397 & 13.241 \\ \text { Debt Ratio } & 21.229 & 4.614 \\ \text { Current Ratio } & 1.673 & 3.866\end{array}$

The Linear Discriminant Functions are Equation 11 and 12:

$$
\begin{aligned}
\text { Bankrupt Score } & =-11.852-9.397(\mathrm{ROA}) \\
& +21.229(\mathrm{DEBT}) \\
& +1.673(\mathrm{CURRENT}) \\
\text { Solvent Score } & =-5.722+13.241(\mathrm{ROA}) \\
& +4.614(\mathrm{DEBT}) \\
& +3.866(\mathrm{CURRENT})
\end{aligned}
$$

The MINITAB results show that there were no misclassifications on the original forty retail firms. To see if there are misclassifications on the second data set of the fifteen retail firms, the above discriminant functions were used for prediction of solvency or bankruptcy. As one can see in Table 12, these discriminant functions seem to be good models to predict bankruptcy. This model is $93 \%$ correct because out of the fifteen retail firms, Liz Claiborne, which is labeled as solvent was predicted to go bankrupt. Liz Claiborne's solvent score was smaller than its bankrupt score. Hence, the function labeled it as insolvent. It should also be noted that its original and revised Altman z-scores were 5.03 and 6.47 respectively, which classified Liz Claiborne as solvent. However, this discriminant function does predict this data set more accurately that both the original and revised Altman $\mathrm{z}$ models. Respectively, they are only 86 and $80 \%$ correct compared to the debt ratio, current ratio and ROA discriminant function, which is $93 \%$ correct.

Table 12. Predictions of the fifteen firms from the $2^{\text {nd }}$ discriminant model

\begin{tabular}{llllllll} 
Test Company & Group & ROA & Debt Ratio & Current ratio & Bankrupt Score & Solvent Score & Prediction \\
\hline WMT & Solvent & $\$ 0.0800$ & $\$ 0.2800$ & 0.88242375 & -5.274114206 & 0.038822220 & 1 \\
URBAN & Solvent & $\$ 0.1200$ & $\$ 0.0000$ & 2.56223176 & -8.736845161 & 5.834251729 & 1 \\
SAKS & Solvent & $\$ 0.0400$ & $\$ 0.1800$ & 2.73551637 & -3.865659680 & 6.133264286 & 1 \\
Stein & & & & & & & \\
Mart & Solvent & $\$ 0.0400$ & $\$ 0.0000$ & 1.93709028 & -9.005474471 & 2.322282493 & 1 \\
Fossil & Solvent & $\$ 0.1800$ & $\$ 0.0100$ & 3.15856777 & -8.061130598 & 8.908563493 & 1 \\
GNC & Solvent & $\$ 0.0500$ & $\$ 0.3700$ & 3.05194805 & 0.623519790 & 8.508781786 & 1 \\
Vitamin & Solvent & $\$ 0.0900$ & $\$ 0.0000$ & 1.82873806 & -9.613079665 & 2.571402916 & 1 \\
ANF & Solvent & $\$ 0.0400$ & $\$ 0.0200$ & 2.11205673 & -8.309189839 & 3.087063054 & 1 \\
ARO & Solvent & $\$ 0.1000$ & $\$ 0.0000$ & 2.29015544 & -8.915522329 & 4.392788552 & 1 \\
Children & Solvent & $\$ 0.0900$ & $\$ 0.0000$ & 3.58333333 & -6.707340678 & 9.329235997 & 1 \\
Sears & Solvent & -0.1469 & 0.1634 & 1.11000000 & -5.145732100 & -2.621915300 & 1 \\
Costco & Solvent & 0.0546 & 0.0810 & 1.13740000 & -8.742657000 & -0.228119000 & 1 \\
Liz & & & & & & & \\
Claiborne & Solvent & -0.1811 & 0.4695 & 1.29270000 & 1.979499300 & -0.956093900 & 0 \\
Circuit & & & & & & & \\
City & Bank & -0.0900 & 0.600 & 1.52000000 & 4.274090000 & 1.731030000 & 0 \\
Finlay & & & & & & & \\
Enterprises & Bank & -0.1891 & 0.8300 & 1.52000000 & 10.088002700 & 1.480066900 & 0
\end{tabular}


The last discriminant function that was formulated consisted of the current ratio and profit margin. This is the smallest discriminant function created for predicting bankruptcy in this study. An analyst does not need a whole lot of explanatory variables in a discriminant function to correctly predict bankruptcy. A smaller function can still produce good predictions. As mentioned before, the current ratio is a company's current assets divided by its current assets. Profit margin is a profitability ratio, which is also called return on sales. It is calculated by dividing a firm's net income by total sales. All of the discriminant functions mentioned in this study have one thing in common, which is their explanatory financial variables all consist of profitability and balance sheet ratios. This is very important because a discriminant function must encompass all types of ratios. A discriminant function that only has one leverage or profitability ratio will not properly predict a firm's financial destiny. A firm might have a negative profitability ratio but strong leverage ratios. Using only on type of financial ratio as an explanatory variable does not tell the analyst the whole financial story about the firm. Below are the discriminant results of the Profit Margin Current Ratio Score.

Discriminant Analysis: Bankruptcy versus Current Ratio, NI/Sales

Linear Method for Response: Bankruptcy

Predictors: Current Ratio, NI/Sales

$\begin{array}{lll}\text { Group } & 0 & 1 \\ \text { Count } & 18 & 22\end{array}$

Summary of classification

$\begin{array}{lll} & \text { True } & \text { Group } \\ \text { Put into Group } & 0 & 1 \\ 0 & 17 & 0 \\ 1 & 1 & 22 \\ \text { Total N } & 18 & 22 \\ \text { N correct } & 17 & 22 \\ \text { Proportion } & 0.944 & 1.000 \\ & & \\ \mathrm{~N}=40 & \text { N Correct }=39\end{array}$

Proportion Correct $=0.975$

Squared Distance Between Groups

$\begin{array}{lll} & 0 & 1 \\ 0 & 0.00000 & 6.54560 \\ 1 & 6.54560 & 0.00000\end{array}$

Linear Discriminant Function for Groups

$\begin{array}{lll} & 0 & 1 \\ \text { Constant } & -2.216 & -4.382 \\ \text { Current Ratio } & 2.041 & 3.471 \\ \text { NI/Sales } & -25.594 & 8.716\end{array}$

Summary of Misclassified Observations

$\begin{array}{llllll} & \text { True } & \text { Pred } & & \text { Squared } & \\ \text { Obs } & \text { Group } & \text { Group } & \text { Group } & \text { Distance } & \text { Probability } \\ 11 * * & 0 & 1 & 0 & 2.260 & 0.385 \\ & & & 1 & 1.323 & 0.615\end{array}$

The Linear Discriminant Functions are Equation 13 and 14:

$$
\begin{gathered}
\text { Bankrupt Score }=-2.216+2.041(\text { CURRENT }) \\
-25.59(\mathrm{PM}) \\
\begin{aligned}
\text { Solvent Score }= & -4.382+3.471(\text { CURRENT }) \\
+ & 8.716(\mathrm{PM})
\end{aligned}
\end{gathered}
$$

Unlike the other two discriminant functions, the profit margin current ratio score produces one misclassification in the original data set of forty retail firms. Observation eleven or Merry Go Round Company was predicted to be solvent even though in reality it went bankrupt. This is because it had a high current ratio of 2.1 and a high profit margin for a bankrupt company of -.01. This resulted in the formula giving Merry Go Round Company a higher solvent score than its bankrupt score. Table $\mathbf{1 3}$ shows all of explanatory ratios and the results of the prediction of all forty retail firms including Merry Go Round Company. One can see that Merry Go Round's ratios are quite different than the other bankrupt retail firms.

After testing the original forty data, the second set of data was tested on this model. According to the second set of data, the current ratio profit margin discriminant function was $93 \%$ accurate. Sears Holdings Company was the only misclassification. The discriminant function predicted Sears to go bankrupt, but in reality Sears is still a solvent retail firm. The reason of this is because Sears has a negative profit margin of -0755 and a low current ratio of 1.11 , compared to the retail average current ratio of 2.13. Table 14 shows the prediction output of the total fifteen retail firms.

The overall results in Table $\mathbf{1 5}$ show the accuracy of three new discriminant functions compared to the BMW model and Altman's original and revised z-score models. One can see that the new discriminant models are more accurate and consistent than Altman's two functions on the original and secondary data sets. 
Table 13. Predictions of forty firms from the $3^{\text {rd }}$ discriminant model

\begin{tabular}{|c|c|c|c|c|c|c|}
\hline Company & Group & C.R. & NI/Sales & Bankrupt Score 0 & Solvent Score 1 & Prediction \\
\hline $\begin{array}{l}\text { Blockbuster } \\
\text { Buffet }\end{array}$ & Bankrupt & 1.024068 & -0.01330 & 0.214523647 & -0.943381651 & 0 \\
\hline $\begin{array}{l}\text { Holdings } \\
\text { Borders }\end{array}$ & Bankrupt & 0.467168 & -0.02130 & -0.717357629 & -2.946110191 & 0 \\
\hline Group & Bankrupt & 0.859261 & -0.04100 & 0.587105764 & -1.756860962 & 0 \\
\hline Lamonts & Bankrupt & 0.740564 & -0.07700 & 1.266229685 & -2.482633401 & 0 \\
\hline $\begin{array}{l}\text { Ames } \\
\text { Linens }\end{array}$ & Bankrupt & 0.950650 & -0.08700 & 1.950954835 & -1.840585535 & 0 \\
\hline $\begin{array}{l}\mathrm{n} \text { things } \\
\text { Shoe }\end{array}$ & Bankrupt & 1.927921 & -0.11000 & 4.534226614 & 1.351053540 & 0 \\
\hline Pavilion & Bankrupt & 1.486199 & -0.02130 & 1.362485127 & 0.590947235 & 0 \\
\hline Tweeter & Bankrupt & 1.367535 & -0.05310 & 1.934180284 & -0.098105702 & 0 \\
\hline Caldor & Bankrupt & 1.097544 & -0.00600 & 0.177652167 & -0.624719309 & 0 \\
\hline $\begin{array}{l}\text { Bradlees } \\
\text { Merry }\end{array}$ & Bankrupt & 0.908835 & -0.23800 & 5.730303697 & -3.301842630 & 0 \\
\hline $\begin{array}{l}\text { Go Round } \\
\text { Movie }\end{array}$ & Bankrupt & 2.086278 & -0.01012 & 2.301103947 & 2.771263776 & 1 \\
\hline $\begin{array}{l}\text { Gallery } \\
\text { Dairy Mart }\end{array}$ & Bankrupt & 0.892562 & -0.09770 & 2.106252494 & -2.135471090 & 0 \\
\hline $\begin{array}{l}\text { Stores } \\
\text { White Hall }\end{array}$ & Bankrupt & 0.772413 & -0.08200 & 1.459202079 & -2.415667929 & 0 \\
\hline Jewelers & Bankrupt & 1.441185 & -0.31000 & 8.659599255 & -2.081605725 & 0 \\
\hline Shells & Bankrupt & 0.276053 & -0.06300 & -0.040153392 & -3.972927297 & 0 \\
\hline Eddie & Bankrupt & 1.355059 & -0.10000 & 3.109075626 & -0.550189859 & 0 \\
\hline Samsonite & Bankrupt & 1.535648 & -0.10000 & 3.477657567 & 0.076634207 & 0 \\
\hline $\begin{array}{l}\text { Penntraffic } \\
\text { Aaron's. }\end{array}$ & Bankrupt & 1.701625 & -0.02020 & 1.774015373 & 1.348277087 & 0 \\
\hline $\begin{array}{l}\text { inc } \\
\text { Big Lots }\end{array}$ & Solvent & 4.466519 & 0.06000 & 5.364526299 & 11.644249180 & 1 \\
\hline $\begin{array}{l}\text { Inc } \\
\text { Dollar }\end{array}$ & Solvent & 1.940959 & 0.04000 & 0.721738155 & 2.703710111 & 1 \\
\hline $\begin{array}{l}\text { General } \\
\text { Dollar }\end{array}$ & Solvent & 1.950988 & 0.05000 & 0.486266798 & 2.825679842 & 1 \\
\hline $\begin{array}{l}\text { Tree } \\
\text { Family }\end{array}$ & Solvent & 2.500938 & 0.07000 & 1.096834634 & 4.908876098 & 1 \\
\hline Dollar & Solvent & 1.508358 & 0.05000 & -0.417141495 & 1.289310324 & 1 \\
\hline Fred’s Inc & Solvent & 2.905405 & 0.02000 & 3.202052432 & 5.876982162 & 1 \\
\hline $\begin{array}{l}\text { Kohl's Corp } \\
\text { Nordstrom, }\end{array}$ & Solvent & 2.028767 & 0.06000 & 0.389072693 & 3.182808975 & 1 \\
\hline Inc & Solvent & 2.567323 & 0.07000 & 1.232326333 & 5.139298286 & 1 \\
\hline $\begin{array}{l}\text { JC Penny } \\
\text { Dillard's, }\end{array}$ & Solvent & 2.406498 & 0.02000 & 2.183782259 & 4.145274288 & 1 \\
\hline $\begin{array}{l}\text { Inc } \\
\text { PriceSmart, }\end{array}$ & Solvent & 2.048135 & 0.03000 & 1.196423081 & 2.988555812 & 1 \\
\hline Inc & Solvent & 1.344037 & 0.04000 & -0.496581101 & 0.631791376 & 1 \\
\hline $\begin{array}{l}\text { Target Corp } \\
\text { Whole }\end{array}$ & Solvent & 1.709335 & 0.04000 & 0.248992036 & 1.899740596 & 1 \\
\hline $\begin{array}{l}\text { Foods } \\
\text { TJX }\end{array}$ & Solvent & 1.653015 & 0.03000 & 0.389983185 & 1.617094334 & 1 \\
\hline Companies & Solvent & 1.627833 & 0.06000 & -0.429233361 & 1.791167469 & 1 \\
\hline Coach Inc & Solvent & 2.448567 & 0.21000 & -2.593215548 & 5.947334705 & 1 \\
\hline PetsMart & Solvent & 1.958188 & 0.04000 & 0.756902021 & 2.763511080 & 1 \\
\hline Tiffany & Solvent & 5.59375 & 0.12000 & 6.129563750 & 16.079826250 & 1 \\
\hline $\begin{array}{l}\text { Ann } \\
\text { Home }\end{array}$ & Solvent & 1.660839 & 0.04000 & 0.150012727 & 1.731412727 & 1 \\
\hline $\begin{array}{l}\text { Depot } \\
\text { Tractor }\end{array}$ & Solvent & 1.548635 & 0.06000 & -0.590876348 & 1.516271433 & 1 \\
\hline Supply & Solvent & 2.364425 & 0.05000 & 1.330091757 & 4.260719740 & 1 \\
\hline Nike & Solvent & 2.854219 & 0.10000 & 1.050061597 & 6.396595200 & 1 \\
\hline American & Solvent & 3.177778 & 0.05000 & 2.990144444 & 7.083866667 & 1 \\
\hline
\end{tabular}


Table 14. Predictions of the fifteen firms from the $3^{\text {rd }}$ discriminant model

\begin{tabular}{|c|c|c|c|c|c|c|}
\hline Test Company & Group & Current ratio & NI/Sales & Bankrupt Score 0 & Solvent Score 1 & Prediction \\
\hline WMT & Solvent & 0.88242375 & 0.04 & $(\$ 1.31)$ & $(\$ 1.01)$ & 1 \\
\hline URBAN & Solvent & 2.56223176 & 0.07 & $\$ 1.10$ & $\$ 5.16$ & 1 \\
\hline SAKS & Solvent & 2.73551637 & 0.02 & $\$ 2.73$ & $\$ 5.33$ & 1 \\
\hline Stein Mart & Solvent & 1.93709028 & 0.17 & $(\$ 2.68)$ & $\$ 3.84$ & 1 \\
\hline Fossil & Solvent & 3.15856777 & 0.11 & $\$ 1.29$ & $\$ 7.58$ & 1 \\
\hline GNC & Solvent & 3.05194805 & 0.06 & $\$ 2.38$ & $\$ 6.77$ & 1 \\
\hline Vitamin & Solvent & 1.82873806 & 0.05 & $\$ 0.17$ & $\$ 2.42$ & 1 \\
\hline ANF & Solvent & 2.11205673 & 0.03 & $\$ 1.31$ & $\$ 3.22$ & 1 \\
\hline ARO & Solvent & 2.29015544 & 0.03 & $\$ 1.69$ & $\$ 3.83$ & 1 \\
\hline Children & Solvent & 3.58333333 & 0.04 & $\$ 3.95$ & $\$ 8.45$ & 1 \\
\hline Sears & Solvent & 1.11000000 & $(0.08)$ & $\$ 1.98$ & $(\$ 1.19)$ & 0 \\
\hline Costco & Solvent & 1.13740000 & 0.02 & $(\$ 0.31)$ & $(\$ 0.29)$ & 1 \\
\hline \multicolumn{7}{|l|}{ Liz } \\
\hline Claiborne & Solvent & 1.29270000 & $(0.11)$ & $\$ 3.32$ & $(\$ 0.88)$ & 0 \\
\hline \multicolumn{7}{|l|}{ Circuit } \\
\hline City & Bankrupt & 1.52000000 & $(0.03)$ & $\$ 1.58$ & $\$ 0.66$ & 0 \\
\hline \multicolumn{7}{|l|}{ Finlay } \\
\hline Enterprises & Bankrupt & 1.52000000 & $(0.14)$ & $\$ 4.53$ & $(\$ 0.35)$ & 0 \\
\hline
\end{tabular}

Table 15. Correctness of altman Z, BMW model, revised altman $\mathrm{Z}$ models and the new discriminant models

\begin{tabular}{lll}
\hline Model & $\begin{array}{c}\text { \% correct on } \\
\text { original 40 }\end{array}$ & $\begin{array}{l}\% \text { correct On } \\
\text { second 15 }\end{array}$ \\
\hline Original & $92.5 \%$ & $86 \%$ \\
BMW & $98 \%$ & $80 \%$ \\
Revised & $100 \%$ & $80 \%$ \\
New Function $1^{*}$ & $100 \%$ & $93 \%$ \\
New Function 2** & $100 \%$ & $93 \%$ \\
New Function 3*** & $98 \%$ & $93 \%$ \\
\hline$*$ Consists of NWC/TA, RE/TA, BE/TL and EBIT/TA as ex- \\
planatory variables; **Consists of ROA, Debt Ratio and \\
Current Ratio as explana-tory variables; ***Consists of Profit \\
Margin and Current Ratio as explanatory variables
\end{tabular}

\section{CONCLUSION}

The retail industry is a big force in the US economy. With the overall retail industry having sales totaling $\$ 4.7$ trillion in 2011 and with a US workforce of 14 million, it remains as one of the most dominant and important industries that effect the US economy. Since the retail industry has such a huge effect on many US suppliers, communities, employees, investors and customers, it should have its own bankruptcy prediction model that can better predict future solvency or bankruptcy. A discriminant model that can foresee the probability of default can be beneficial to those who are affected by retail bankruptcy. Foreseeing bankruptcy can get managers attention to fix current financial problems.

Other industries such as technology, chemicals, telecom, banking, energy and are also profitable and affect the US economy as well. Discriminant functions for each separate industry should be used to predict bankruptcy. When using MDA, it is best to create separate discriminant functions using a recent sample from the industry in question. For example, it is not reasonable to assume that financial ratios of a steel company facing bankruptcy are the same as for a retail discount corporation. These two industries have different capital structures and different profitability averages. Separate discriminant scores should be developed for each industry.

The analysis has concluded that the newly formulated discriminant functions in this study predicted bankruptcy and solvency for retail firms better than the Begley, Ming and Watts model and Altman's original and revised discriminant models. The BMW model or the reestimated Altman model had one misclassification on the original forty data sample and 3 misclassifications on the second data sample, making it 98 and $80 \%$ accurate respectively. The original Altman z-score had three misclassifications on the original forty data, making it $92.5 \%$ accurate. The revised Altman z-score model was $100 \%$ accurate on the original forty data firms and the first two new discriminant retail models were also $100 \%$ accurate on the forty retail firms, while the last new discriminant model using profit margin and the current ratio was only $97.5 \%$ accurate. The three newly derived discriminant models were all $93.33 \%$ correct on the test data (second set of data) while the original and revised Altman z-score models were only 86.67 and $80 \%$ correct, respectively on the test data. Hence, the newly formulated discriminant models that were derived in this study should be used instead of Altman's discriminant models when predicting retail probability of default. 


\section{REFERENCES}

Altman, E.I., 1968. Financial ratios, Discriminant analysis and the prediction of corporate bankruptcy. J. Finance 23: 589-609. DOI: 10.1111/j.15406261.1968.tb00843.x

Altman, E.I., 2000. Predicting financial distress of companies: Revisiting the Z-score and zeta models. J. Finance.
Begley, J., J. Mingv and S. Watts, 1996. Bankruptcy classification errors in the 1980s: An empirical analysis of Altman's and Ohlson's models. Rev. Account. Stud., 1: 267-84. DOI: 10.1007/BF00570833 